\title{
Laminar Gas Jets in High-Temperature Atmospheres
}

\author{
M. Sánchez-Sanz ${ }^{1, *}$, A. L. Sánchez ${ }^{1}$, A. Liñán ${ }^{2}$ \\ ${ }^{1}$ Area de Mecánica de Fluidos, Universidad Carlos III de Madrid, 28911 Leganés, Spain \\ ${ }^{2}$ E. T. S. I. Aeronáuticos, Pl. Cardenal Cisneros 3, 28040 Madrid, Spain
}

Email: mssanz@ing.uc3m.es

\begin{abstract}
Numerical and asymptotic methods are used to describe the structure of low-temperature laminar gas jets discharging into a hot atmosphere of the same gas in the limit of small jet-to-ambient temperature ratios $\varepsilon=T_{j} / T_{o} \ll 1$. The boundary-layer approximation is used to describe the slender steady solution that emerges for moderately large values of Reynolds number $R e=\rho_{o} U_{j} a / \mu_{o}$, where $U_{j}$ represents the exit jet velocity, $a$ is its characteristic transverse dimension (the initial radius for the round jet and the initial half-width for the planar jet) and $\rho_{o}$ and $\mu_{o}$ are the ambient values of density and viscosity. In the limit $\varepsilon \rightarrow 0$, heat conduction cannot modify significantly the temperature in the cold gas, leading to a two-region flow structure consisting of a neatly defined unperturbed cold jet for $r<r_{f}(x)$, where $T=T^{\prime} / T_{o}=\varepsilon$ and $u=U^{\prime} / U_{j}=1$, surrounded by a hot gas. These two region are separated by a transition layer where $T-\varepsilon \sim \varepsilon$ and $1-u \ll 1$.

In planar jets, axial convection and radial transport balance in the surrounding outer gas in a region whose radial extent increases continuously with downstream distance according to $(r-a) \sim \sqrt{x}$. The same balance is found in the thickening transition layer separating the outer region from the unperturbed core, which remains centered at $r=a$, with a thickness that increases continuously with distance according to $r-a \sim \varepsilon^{(1+\sigma) / 2} \sqrt{x}$. Both regions merge at distances $x \sim \varepsilon^{-(1+\sigma)}$ Rea where $T-\varepsilon \sim 1-u \sim \varepsilon$ at the jet axis. In this far field, transverse gradients of temperature and velocity are very small and, consequently, large distances of order $x \sim \varepsilon^{-2} R e a$ are required to achieve axial values of $T$ and $1-u$ of order unity.

In round jets, a thickening layer of very low density gas surrounds initially the jet front, which remains at $r=a$, much like the planar jet. However, for $x \gg$ Rea axial convection becomes negligible and a quasi-steady balance settles outside the jet as the mass emerging from the jet decreases, meanwhile the front remains at $r=a$. At distances $x \sim \varepsilon^{-(1-\sigma)}\left(\log \varepsilon^{-1}\right)^{2} R e a$ the front starts to move radially towards the axis as the cold gas is ablated from the cold jet surface. The front, that thickens as $r-r_{f} \sim \varepsilon^{\sigma} \log \sqrt{x}$, reaches the center of the jet at $x \sim \varepsilon^{-1} \log \varepsilon^{-1}$ Rea.
\end{abstract}

\section{REFERENCES}

1. Abramowitz M, Stegun A. Handbook of mathematical functions. New York: Dover, 1965

2. Tarifa C S, Crespo A, Fraga E. A theoretical model for the combustion of droplets in super-critical conditions and gas pockets. Astronautica Acta, 1971; 17: 685-692

3. Pai S I. Axially symmetrical jet mixing of a compressible fluid. Quar Appl Math, 1971; 10: 141-148

4. Pai S I. Fluid Dynamics of Jets. Toronto: Van D Nostrand Company, 1954 